\title{
Künstlersozialabgabe und Laienchor
}

Landessozialgericht Berlin-Brandenburg, Urteil vom 27. Oktober 2010 - L 1 KR 48/09

1. Wöchentliche Proben, sechs bis 12 Chorsamstage im Jahr und jährliche Chorfahrten sollen nicht lediglich der Vorbereitung von drei Konzerten dienen, sondern haben einen eigenen, sich nach Anschauung der Mitglieder des Chores aus sich selbst heraus ergebenden Wert, nämlich den, gemeinsam das Hobby des Chorsingens zu betreiben; demgegenüber sind die Konzerte, auch wenn sie einen Höhepunkt im Vereinsleben darstellen mögen, nicht von solcher Bedeutung, dass sie die Tätigkeit des Chores des Klägers überwiegend iSv § 24 KSVG idF des Wachstums- und Beschäftigungsförderungsgesetzes vom 25 . September 1996 prägen.

2. Wurden im Zeitraum von Juli 2001 bis zum 31. Dezember 2004 nicht mehr als drei Veranstaltungen zur Aufführung künstlerischer Werke durchgeführt, so liegt auch nach § 24 Abs. 2 KSVG idF des Änderungsgesetzes vom 13. Juni 2001 nur eine gelegentliche Erteilung von Aufträgen vor. (Leitsätze der Redaktion)

\section{Tenor}

- Der Gerichtsbescheid des Sozialgerichts Berlin vom 17. Dezember 2008 wird geändert.

Die Bescheide der Beklagten vom 16. Dezember 2004, 22. Dezember 2004 und vom 1. April 2005 in der Gestalt des Widerspruchsbescheides vom 2. August 2005 sowie der Bescheid vom 25. Oktober 2005 werden aufgehoben.

Die Beklagte trägt die außergerichtlichen Kosten der Klägerin für beide Rechtszüge.

Die Revision wird nicht zugelassen.

Der Streitwert wird für beide Rechtszüge auf 5.000,00€ festgesetzt.

\section{Tatbestand}

Im Streit zwischen den Beteiligten ist, ob der Kläger als Unternehmer der Abgabenpflicht zur Künstlersozialversicherung von 1999 bis 2004 unterliegt.

Der im Jahre 1973 gegründete Kläger betreibt den H.-Chor in Berlin, der seit dem Jahre 1953 besteht. Nach seiner Satzung ist der Kläger Träger des H.-Chores Berlin. Ihm obliegt die Schaffung und Erweiterung von künstlerischen Wirkungsmöglichkeiten des Chores und die Durchführung von Konzertveranstaltungen und Konzertreisen, wobei ihm die Pflege des Werkes von $\mathrm{H}$. ein besonderes Anliegen ist, Die erforderlichen Mittel werden u. a. durch Einträge aus Veranstaltungen und Honoraren aufgebracht.

Die Beklagte forderte im Sommer 2004 den Kläger auf, ihren Fragebogen zur Abgabepflicht nach dem Künstlersozialversicherungsgesetz - KSVG - vorzulegen, was am 7. September 2004 erfolgte. Darin war angegeben, dass der Kläger einen Chor betreibt und in diesem Zusammenhang gelegentlich, nämlich zirka dreimal jährlich, Aufträge an selbständige Künstler oder Publizisten erteilt. Dabei handele es sich um Chorleiter,
Instrumentalsolisten, Musiker, Komponisten und Sänger. Diese würden von Dienst- oder Werkverträgen selbständig erbracht. Die Abgabenpflicht sei dem Kläger seit 2004 bekannt.

Mit Schreiben vom 12. Oktober 2004 vertrat die Beklagte die Auffassung, es läge Abgabepflicht nach dem KSVG vor, und bat um Angaben über die zurückliegenden Jahre. Daraufhin teilte der Kläger mit Schreiben vom 12. November 2004 mit, dass in den Jahren 1999, 2000, 2001, 2002 und 2003 jeweils drei Konzerte jährlich stattgefunden hätten. Er verwies darauf, dass Gemeinnützigkeit vorläge und Konzerte nur gelegentlich, nämlich unter viermal jährlich, stattfänden.

Mit Bescheid vom 10. Dezember 2004 setzte die Beklagte die Künstlersozialabgabe - KSA - für die Jahre 1999 bis 2003 und die Vorauszahlungen für Januar und Februar 2004 auf insgesamt $8.609,58 €$ aufgrund einer Schätzung fest. Mit Bescheid vom 22. Dezember 2004 stellte sie die Abgabepflicht des Klägers dem Grunde nach fest. Der Kläger ermittelte einen Meldebogen für das Jahr 2004, daraufhin setzte die Beklagte mit Bescheid vom 1. April 2005 die KSA für das Jahr 2004 auf 483,75 € fest und verfügte monatliche Vorauszahlungen in Höhe von $54,38 €$. Gegen all diese Bescheide erhob der Kläger Widerspruch, den die Beklagte mit Widerspruchsbescheid vom 2. August 2005 zurückwies.

Hiergegen hat sich die am 2. September 2005 beim Sozialgericht Berlin erhobene Klage gerichtet, zu deren Begründung der Kläger ausgeführt hat, er betreibe einen Laienchor, öffentliche Ausführungen seien nur von untergeordneter Bedeutung. Im Vordergrund stünde das Vereinsleben, bestehend aus wöchentlichen Übungen, einem jährlichen Chorwochenende, so genannten Chorsamstagen alle ein bis zwei Monate unter Einbeziehung der Familien der Chorsänger, einem jährlichen Sommerfest und einer jährlichen Weihnachtsfeier. Die Chormitglieder erhielten keine Honorare. Lediglich bei jeweils drei öffentlichen Auftritten im Jahr würden fremde Künstler engagiert, die Honorare erhielten.

Die Beklagte hat mit Bescheid vom 25. Oktober 2005 die KSA für die Jahre 1999 bis 2004 neu festgesetzt und die Beitragsforderung auf insgesamt $2.296,63 €$ herabgesetzt. Sie hat 
im Übrigen die Auffassung vertreten, dem Grunde nach seien auch gemeinnützige Vereine abgabepflichtig. Satzungsgemäß und praktisch sei der Schwerpunkt der Tätigkeit des Klägers das öffentliche Auftreten des Chores. Auch habe dieser im Jahr 2005 Mittel aus der Chorförderung des Landes Berlin erhalten, die nur an Konzert- und Oratorienchöre vergeben würden, die zusammen mit professionellen Partnern Aufführungen auf hohem Niveau realisierten.

Das Sozialgericht hat mit Gerichtsbescheid vom 17. Dezember 2008 die Klage abgewiesen und zur Begründung im Wesentlichen ausgeführt, der Chor des Klägers trete öffentlich auf und dies sei sein überwiegender Zweck. Seine Aufnahme in den Kreis der vom Land Berlin geförderten Chöre mache nach außen hin deutlich, dass sein Zweck überwiegend darauf gerichtet sei, künstlerische Leistungen auf hohem Niveau öffentlich aufzuführen und darzubieten. Gegen diesen dem Prozessbevollmächtigten des Klägers am 15. Januar 2009 zugestellten Gerichtsbescheid richtet sich die Berufung vom 10. Februar 2009, mit der das Vorbringen erster Instanz wiederholt und vertieft wird.

\section{Der Kläger beantragt,}

den Gerichtsbescheid des Sozialgerichts Berlin vom 17. Dezember 2008 zu ändern und die Bescheide der Beklagten vom 16. Dezember 2004, 22. Dezember 2004 sowie vom 1. April 2005 in der Gestalt des Widerspruchsbescheides vom 2. August 2005 und den Bescheid vom 25. Oktober 2005 aufzuheben.

Die Beklagte beantragt,

die Berufung zurückzuweisen.

Sie hält den angefochtenen Gerichtsbescheid für zutreffend.

\section{Entscheidungsgründe}

Über die zulässige Berufung konnte der Berichterstatter ohne mündliche Verhandlung entscheiden (§§ 124 Abs. 1, 155 Sozialgerichtsgesetz - SGG -). Die Berufung ist begründet. Der Kläger unterliegt nicht der Abgabepflicht bei der Beklagten, so dass die entgegenstehenden Bescheide der Beklagten und der sie bestätigende Gerichtsbescheid ihn in seinen Rechten verletzten und daher aufzuheben waren.

Nach § 24 KSVG in der Fassung des Wachstums- und Beschäftigungsförderungsgesetzes vom 25. September 1996 waren Unternehmen in der Zeit vom 1. Januar 1997 an als Betreiber eines Chores nur dann zur Abführung der KSA verpflichtet, wenn der Zweck des Unternehmens überwiegend darauf gerichtet ist, künstlerische oder publizistische Werke oder Leistungen öffentlich aufzuführen oder darzubieten. Diese die Abgabepflicht deutlich einschränkende Voraussetzung erfüllte der Kläger nicht. Der Unternehmenszweck eines Musikvereins ist nur dann „überwiegend" darauf gerichtet, künstlerische Werke oder Leistungen öffentlich aufzuführen oder darzubieten, wenn der Schwerpunkt der Tätigkeit nach der Vereinssatzung und der Praxis des Chores auf dem öffentlichen Auftreten einschließlich der zugehörigen Probenarbeiten liegt und demgegenüber andere - nichtkommerzielle - Zwecke wie z. B. die Freizeitgestaltung, die Pflege eines Hobbys, die Freude am gemeinsamen Musizieren, der regelmäßige gesellschaftliche Kontakt in der Gruppe sowie die Aufrechterhaltung und Förderung des Vereinslebens nur untergeordneten Charakter haben. Diese Voraussetzungen treffen auf den vom Kläger betriebenen Chor nicht zu. Seine Mitglieder sind Laien, die ein Hobby pflegen. Sie üben die Tätigkeit im Chor weder haupt- noch nebenberuflich aus. Die öffentlichen Auftritte des Chores finden nur dreimal jährlich statt. Lediglich für sie werden Vereinsfremde für ein Honorar engagiert. Es widerspricht einer natürlichen Betrachtungsweise, dass wöchentliche Proben, sechs bis 12 Chorsamstage im Jahr und jährliche Chorfahrten lediglich der Vorbereitung von drei Konzerten dienen sollen. Sie haben zur Überzeugung des Gerichts vielmehr einen eigenen, sich nach Anschauung der Mitglieder des Chores aus sich selbst heraus ergebenden Wert, nämlich den, gemeinsam das Hobby des Chorsingens zu betreiben. Demgegenüber sind die Konzerte, auch wenn sie einen Höhepunkt im Vereinsleben darstellen mögen, nicht von solcher Bedeutung, dass sie die Tätigkeit des Chores des Klägers überwiegend prägen (vgl. hierzu Bundessozialgericht - BSG -, Urteil vom 20. November 2009 - B 3 KS 5/07 R -, zitiert nach juris, Nr. 22, 23).

Auch ab 30. Juni 2001 bestand keine Abgabepflicht. Durch das KSVG-Änderungsgesetz vom 13. Juni 2001 hat der Gesetzgeber in $\S 24$ Abs. 1 Satz 1 Nr. 2 und 3 KSVG jeweils den Halbsatz "Abs. 2 bleibt unberührt" angefügt und Abs. 2 Satz 2 neu gefasst, wonach bei nicht mehr als drei Veranstaltungen jährlich keine Abgabepflicht besteht. Darüber hinaus wurde dort ein Satz 3 angefügt, wonach Abs. 1 Nr. 2 Satz 1 nicht für Musikvereine gilt, soweit für sie Chorleiter oder Dirigenten regelmäßig tätig sind. Dadurch sollten die Musikvereine als Träger von Laienchören negiert werde, wenn Aufträge an selbständige Chorleiter und Dirigenten und das Tatbestandsmerkmal der "nicht nur gelegentlichen“ Auftragserteilung außer Betracht zu bleiben haben (vgl. BSG, Urteil vom 20. November 2008, Nr. 25).

Da der Kläger in den streitigen Kalenderjahren nicht mehr als drei Veranstaltungen durchgeführt hat, in denen künstlerische Werke aufgeführt wurden, liegt auch im Zeitraum von Juli 2001 bis zum 31. Dezember 2004 nach § 24 Abs. 2 KSVG neuer Fassung nur eine gelegentliche Erteilung von Aufträgen vor.

Die Kostenentscheidung folgt aus § 197 a SGG iVm § 154 Abs. 1 VwGO; der Beschluss über den Streitwert aus $\S 52$ GKG. Es wurde auch über die Abgabepflicht dem Grunde nach auf Dauer gestritten, so dass der Regelstreitwert des Absatzes 2 dieser Norm und nicht die (niedrigere $=2.296,63 €$ ) Höhe der streitigen Abgabeforderungen maßgeblich ist.

Die Revision war nicht zuzulassen (§ 160 Abs. 2 SGG). (Entscheidung von der Redaktion bearbeitet.) 